\title{
Effect of changing of the parameters of the cable network of monitoring systems of high- rise buildings on the basis of string converters on their operability
}

\author{
Nikolay Gusev ${ }^{1}$, Larisa Svatovskaya ${ }^{2}$, and Alexandr Kucherenko ${ }^{1, *}$ \\ ${ }^{1}$ Military Space Academy named after A.F. Mozhaysky, Zhdanovskaya st.13, St. Petersburg, 197198, \\ Russia \\ ${ }^{2}$ Emperor Alexander I St. Petersburg State Transport University (PGUPS), Moskovsky pr.9, St. \\ Petersburg, 190031, Russia
}

\begin{abstract}
The article is devoted to the problem of improving the reliability of monitoring systems for the technical conditions of high-rise buildings. The improvement is based on string sensors with an impulsed excitation method ensuring the maximum signal-to-noise ratio at their output. The influence of the parameters of the monitoring system on the shape of the excitation impulses of the string, and, consequently, on the amplitude of the string vibration of the string converter is also considered in the article. It has been experimentally proved that the parameters of the excitation impulses of the string converters. The article presents the results of the experiments showing the effect of the fronts duration of the excitation impulses on the amplitude of the oscillations of the strings. The influence of the fronts duration of the excitation impulse with the frontal lengths up to $0.5 \mathrm{~ms}$ is studied at the excitation impulse duration not exceeding 0.5 times the duration of natural oscillation periods of the string. The experimental data are compared with the theoretical ones and hypotheses explaining their difference are advanced. The article suggests some methods of reducing the influence of the cable-switching equipment system parameters on the amplitude of string oscillations. The possibilities of improving the reliability of the systems developed on the basis of string sensors with an impulsed excitation method and used for monitoring the technical conditions of the high-rise buildings are proposed.
\end{abstract}

\section{Introduction}

In the practice of monitoring the stress-strain state of building structures and soil bases of buildings and construction throughout the whole period of their operation the long-term monitoring systems [1-3], and the systems for monitoring the state of high-rise buildings [4-10], have been widely applied.

\footnotetext{
*Corresponding author: kucher_90@bk.ru
} 
In the terminology of the Ministry of Emergency Situations of the Ministry of Emergency Situations [11-13], such systems were called SMES (structured systems for monitoring and managing the engineering systems of buildings and structures). In hydraulic engineering such systems have received the name ASDC - automated systems of the diagnostic control [14-17].

ASDC and SMES systems, as a rule, are developed on the basis of the string converters. In addition to primary string converters, the SMES and ASDC systems include: switching and secondary equipment (specialized period-meters), as well as the cable network [17].

The method of measurement by using string converters is based on the dependence of the frequency $\boldsymbol{\omega}$ (or period $\boldsymbol{T}$ ) of the frequency resonator (string) oscillation on the string extension stress which in each particular string converter is proportional to the measured parameter $\boldsymbol{P}$ (linear displacement, pressure, temperature, etc.).

To excite the string vibrations of the string measuring transducers with impulse excitation (STMS) electromagnetic coils are used. When reeding an excitation impulse of a rectangular shape of standard amplitude and duration to the terminals, an excitation impulse with a certain amplitude $\boldsymbol{F}$ and duration $\tau_{\boldsymbol{u}}$ is generated in the coil. The force impulse excites the damped oscillations of the string $\boldsymbol{y}$ by "pinch" that induce $\boldsymbol{e}$, the frequency $\boldsymbol{\omega}$, or the period $\boldsymbol{T}$ in the electromagnetic coil $\boldsymbol{e}$, which can be measured up to some preassigned value of the amplitude determined by interference level corresponding to the minimum value $\boldsymbol{y}_{\min }$ string oscillation amplitude. The maximum value of the amplitude of the string oscillations $\boldsymbol{y}_{\text {мах }}$ is limited by the design gap between the string in the unexcited state and the core of the electromagnetic coil of excitation $\delta$. The value of the gap for commercially available STMS lies in the range $0.35 \div 0.50 \mathrm{~mm}$ [17].

Each STMS has an individual calibration, as shown in Fig. (1), produced with the excitation of string vibrations by standard rectangular impulses, their parameters being defined in control documents [17]. The stated parameters of the excitation impulses should provide the value of the amplitude of the damped oscillations of the string in the range from $\boldsymbol{y}_{\text {мах }}$ to $\boldsymbol{y}_{\min }$ for the entire measurement interval $\left(\boldsymbol{t}_{\text {изм }}\right)$. These conditions ensure stable oscillation of the STMS strings and reliable operation of the SMES and ASDC systems.

From the theory of oscillations [18-22] it is known that when an oscillating system is acted upon by a impulse the duration of which is equal to or multiple the system oscillation period, there appears the probability that the system oscillations will not arise or will have a negligible amplitude. Comparative analysis of the excitation impulses duration $\left(\tau_{u}\right)$ of serially produced secondary equipment for STMS [23], the values of which lie in the range from 300 to $700 \mathrm{mks}$, with the duration of the oscillations periods of the STMS (T) strings in the operating range of the measured parameters, 400 to $2000 \mathrm{mks}$ [23], showed that the range of durations $\tau_{u}$ by $25 \div 30 \%$ overlaps the operating range of the values of the oscillation periods of the strings $T$, as shown in Fig. (1).

This means that in the mentioned range of the oscillation period of the STMS string, the values of its amplitude and, consequently, the EMF excitation induced in the electromagnetic coil will fall, which will lead to the decrease in the "signal/noise" ratio at the output of the STMS and the instability of its operation. 


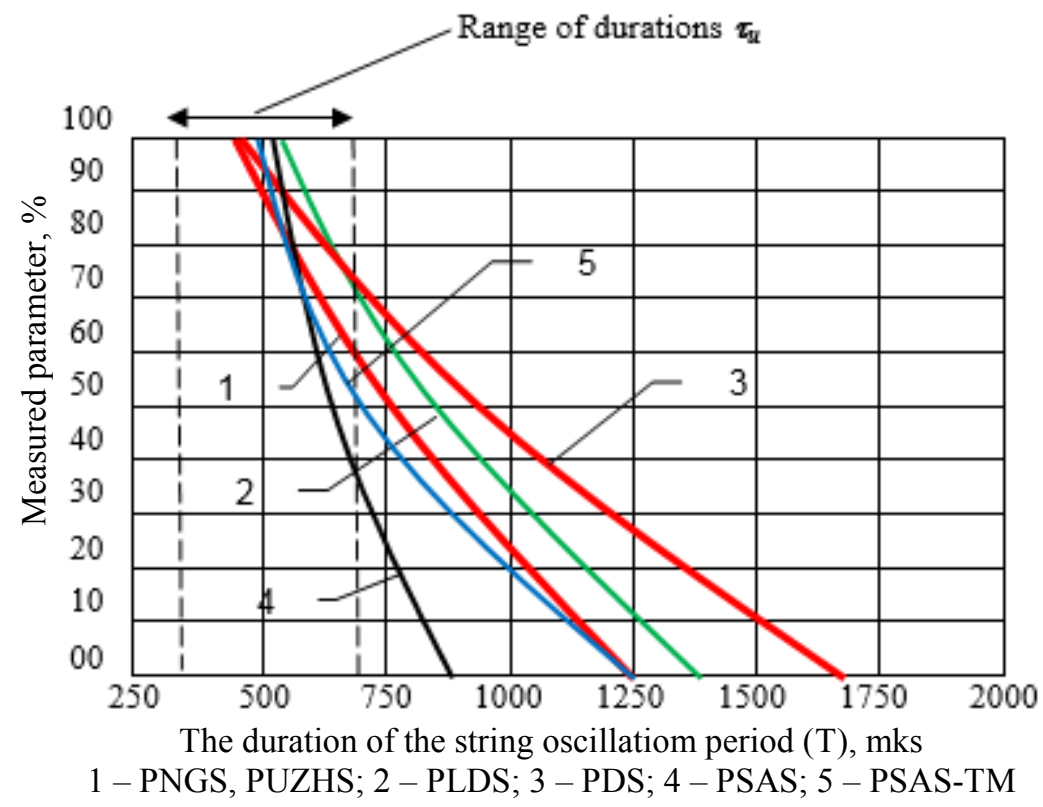

Fig. 1 Dependence of measured quantities on the period of string vibration for string converters with impulsed excitation of string vibrations.

The studies have shown [22] that the parameters of the vibration of the STMS string depend on the amplitude $\boldsymbol{F}$ and the duration of the excitation impulse of the string. The dependence of the string vibration amplitude on the duration of the excitation impulse for various types of STMS is presented in Fig. (2).

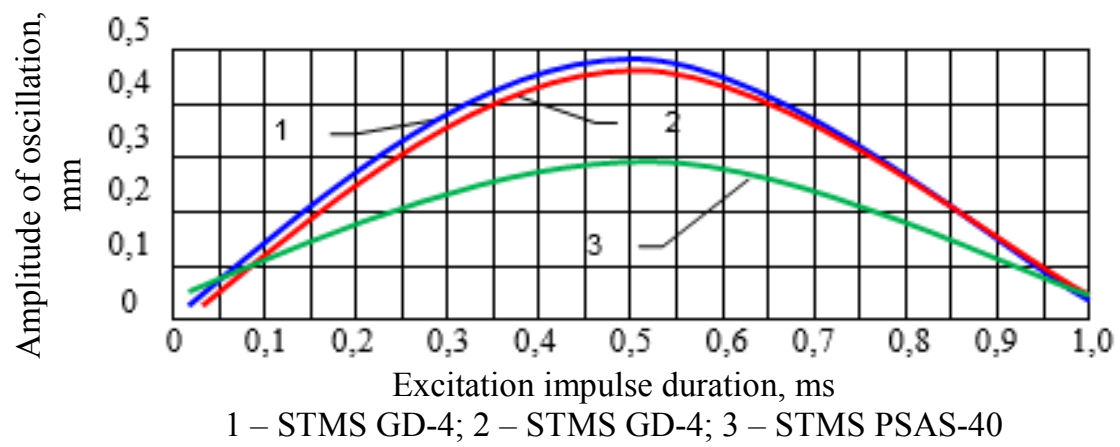

Fig. 2 Dependence of the vibration amplitude of the STMS string on the duration of the excitation impulse.

The invariance of the (standard) values of the parameters of the amplitude $\boldsymbol{F}$ and the duration $\tau_{u}$ of the string excitation impulse with a change in the frequency $\boldsymbol{T}$ of oscillations $\boldsymbol{y}$ of the string in the operating frequency range of the STMS results in the sharp decrease of the string oscillations amplitude. This defect was eliminated in subsequent developments of the secondary equipment with automatic correction of the parameters of the amplitude $\boldsymbol{F}$ and the duration $\tau_{u}$ of the string oscillations impulse as a function of the value of $\boldsymbol{T}$ [22]. 
Experience of the SMES exploitation showed that the string vibration amplitude of each specific standard sensor in the systems of the high-rise buildings monitoring, even when adjusting the parameters of the amplitude $\boldsymbol{F}$ and the duration $\tau_{u}$ of the excitation impulse, differed, and these differences tended to change with time.

The authors put forward the hypothesis that the excitation impulse parameters change while passing through communication cables and switching equipment.

When calibrating the STMS, the secondary equipment is connected directly to the transducer and of rectangular form is performed with a rectangular excitation calibration [24]. However, on real objects in the SMES or ASDC STMS systems, string converters are connected to the secondary equipment by means of cables, for example, CRHD $2 \times 1.5$ (TC 16.505.065-75) and switching equipment, as it is regulated in the normative and technical documentation for STMS. The cable CRHD $2 \times 1.5$ has a capacitance $\boldsymbol{C}$ (the capacitance of 1 $\mathrm{m}$ of cable is not more than $150 \mathrm{pF}$ ) and the electrical resistance of the conductors $\boldsymbol{R}$ (no more than $15 \mathrm{Om}$ per $1 \mathrm{~km}$ of cable length). Consequently it is an integrating circuit. When passing through it a rectangular excitation impulse distorts its shape, which is expressed in the formation of the front and back fronts with a duration of $\tau_{f}$, caused by the time constant of the chain $\boldsymbol{t}=\boldsymbol{R} \boldsymbol{C}$. For normal operation conditions of the CRHD cable $2 \times 1.5$, the value of the chain time constant on the real objects does not exceed $0.1 \div 2.5 \mathrm{mks}$, which is incommensurably small in comparison with the excitation impulse durations (from 300 to $700 \mathrm{mks}$ ) and does not significantly change the process of string oscillations excitation.

However, during operation, the normalized values of $\boldsymbol{C}$ and $\boldsymbol{R}$ of the cable and the contact resistance $\boldsymbol{R}_{\boldsymbol{n} \boldsymbol{k} \boldsymbol{s}}$ of the switching equipment change as a result of aging or external influences and can increase tens or even hundreds times, which results in the significant changes in the shape of the excitation impulse.

The main factors affecting the capacity of the cable are: electrical aging, oxidation, thermal aging and humidification. The investigations carried showed that, with a constant resistance of the cable veins, the formation of the excitation impulse fronts, with the duration $\tau_{f}$, commensurable with the duration $\tau_{u}$, takes place with the increase of the cable capacity CRHD $2 \times 1.5$ tens times, which is quite probable in practice under the influence of the actual parameters of the influencing factors.

The resistance of the cable line consists of the resistance of the cable cores and the contact resistance of the switching equipment of the SMES system.

The most significant contribution to the total resistance of the system "cable line switching equipment" is made by the contact resistance of the commuting equipment, which can increase tens or even hundreds times during corrosion or oxidation of contacts of switching equipment. Thus, during the operation of the cable network and the switching equipment of the SMES systems, their parameters change within the limits that significantly affect the time constant of the circuit and the formation of the fronts of the excitation impulses that approximate its shape to the trapezoidal.

The purpose of this investigation was to state the regularities of the string vibration amplitude change of the string converters used in the systems for high-rise building monitoring. The change is caused by the distortion of the excitation impulse shape. The influence of the process an the efficiency of the monitoring systems has also been investigated.

\section{Materials and methods}

The amplitude $\boldsymbol{y}_{\boldsymbol{n}}$ of the oscillation of the n'th string harmonic excited by a trapezoidal impulse depends on the coefficient of the n'th harmonic $\left(\xi_{\mathrm{n}}\right)$. The coefficient $\xi_{\mathrm{n}}$ depends on the duration of impulse excitation $\tau_{\boldsymbol{u}}$, the duration of its fronts $\tau_{f}$, as well as the circular frequency of the nth harmonic $\omega_{n}$, and has the form [22]: 


$$
\xi_{n}=\frac{2}{\omega_{n} \tau_{f}} \sin \frac{\omega_{n} \tau_{f}}{2} \sin \frac{\omega_{n} \tau_{u}}{2}
$$

Fig. (3). shows the theoretical dependence of the amplitude $\boldsymbol{y}_{\boldsymbol{1}}$ of the first string harmonic vibration on $\tau_{u}$ and $\tau_{f}$ STMS (PLDS -150) at $\boldsymbol{T}_{\boldsymbol{1}}=1.0 \mathrm{~ms}$.

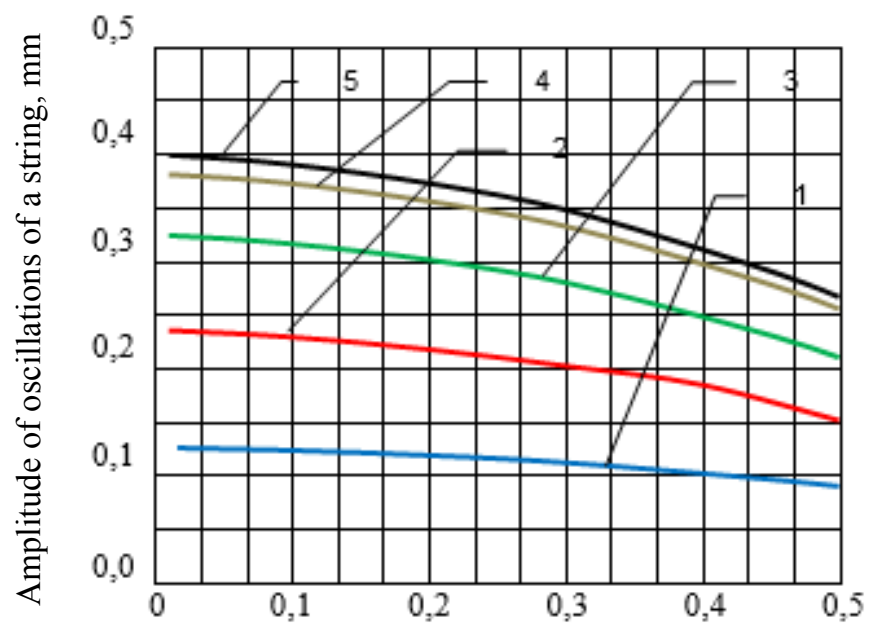

The duration of the edge of the excitation impulse front, $\mathrm{ms}$ $1-\tau_{u}=0,1 \mathrm{~T}_{1} ; 2-\tau_{u}=0,2 \mathrm{~T}_{1} ; 3-\tau_{\boldsymbol{u}}=0,3 \mathrm{~T}_{1} ; 4-\tau_{\boldsymbol{u}}=0,4 \mathrm{~T}_{1} ; 5-\tau_{\boldsymbol{u}}=0,5 \mathrm{~T}_{1}$

Fig. (3). - Dependence of the first harmonic amplitude $(n=1)$ of the string vibration on the duration of the excitation impulse front $\tau_{f}$ for the STMS (PLDS -150) for various values of $\tau_{u}$ at $\boldsymbol{T}_{\boldsymbol{l}}=1.0 \mathrm{~ms}$.

The investigations were carried out by using standard sensors of the PLDS-150 type with the parameters specified in the standards [14, 17], and according to the standard procedure $[23,25]$. The devices for recording static and dynamic displacements (DRSDFD) as the secondary equipment were used. They have the following main parameters:

The parameters of the request impulse (excitation) with a smooth adjustment:

$$
\begin{array}{ll}
\text { voltage } & 135 \div 165 \mathrm{~V}, \\
\text { durability } & 0,25 \div 1,00 \mathrm{~ms}, \\
\text { repetition period } & \text { not less than } 2 \mathrm{~s} .
\end{array}
$$

Parameters of STMS (PLDS-150):

constructive gap $\boldsymbol{\delta} \quad 0,4 \mathrm{~mm}$

output period range $\quad 450 \div 1250 \mathrm{mks}$

Experimental conditions:

$$
\begin{array}{ll}
\text { output cable } & \text { such as CRHD 2x1,5 } \\
\text { ambient temperature } & +20^{\circ} \mathrm{C} \\
\text { recording equipment } & \text { oscilloscope S1-96M }
\end{array}
$$

The experiment was carried out at five values of the impulse duration in excitation $\tau_{u}$ in the range from $0.1 T_{l}$ to $0.5 T_{l}$ in steps of $0.1 T_{l}$ and with a front duration change $\tau_{\phi}$ in the range from 0.0 to $0.5 T_{l}$ in steps of $0.1 T_{l}$ at $T_{l}=1.0 \mathrm{~ms}$. The amplitude of the excitation impulse ensured the maximum value of the string vibration amplitude $\boldsymbol{y}_{\text {мах }} \leq \boldsymbol{\delta}$ at a value of $\boldsymbol{T}_{\boldsymbol{1}}=1.0 \mathrm{~ms}$ at an impulse voltage of $145.5 \mathrm{~V}$.

The shape of the excitation impulse obtained during the experiment is shown in Fig. 4 


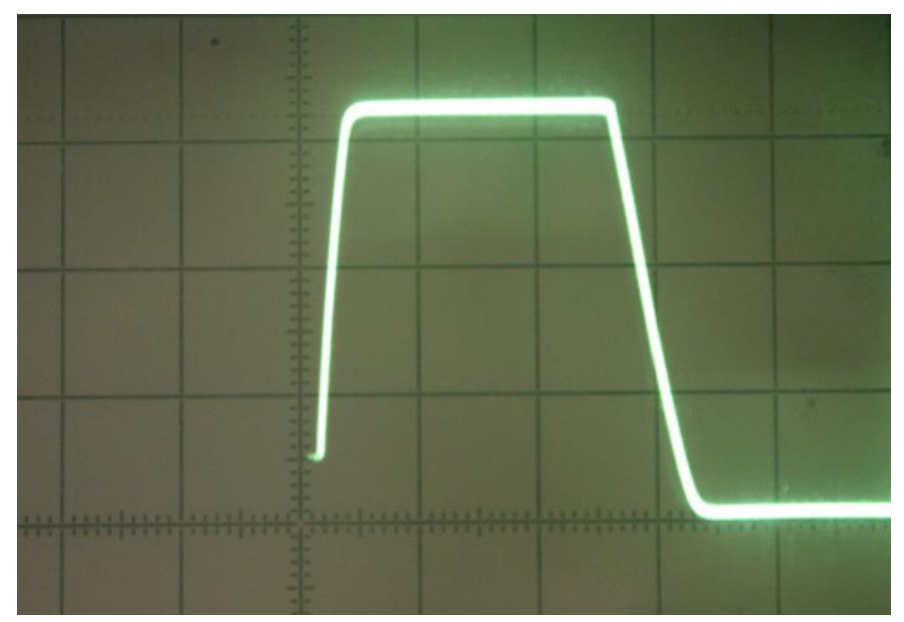

Fig. 4 The shape of the excitation impulse at $\tau_{u}=0,5 T_{l}$ and $\tau_{f}=0,1 T_{l}$.

\section{Results}

The results of the experiment are presented on Fig. 5

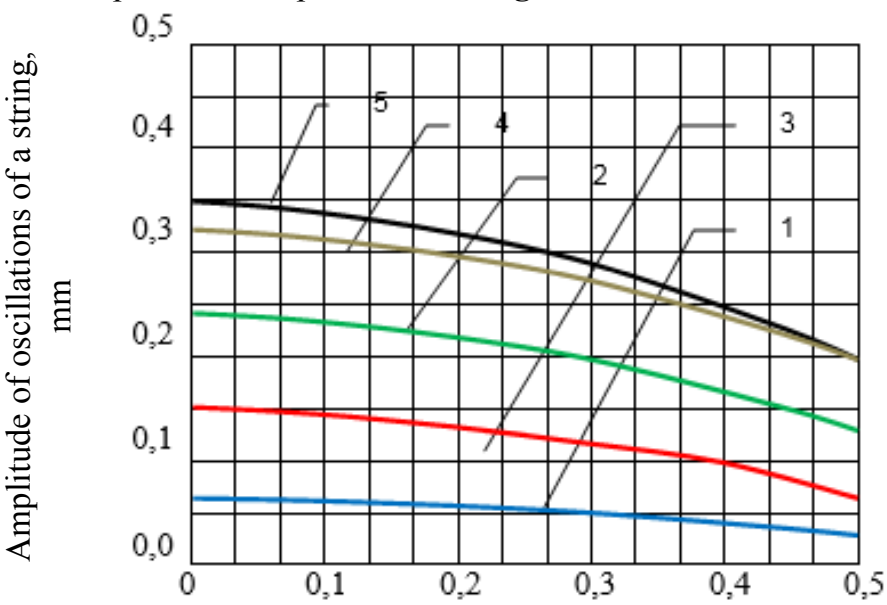

The duration of the excitation impulse front, $\mathrm{ms}$

$1-\tau_{u}=0,1 \mathrm{~T}_{1} ; 2-\tau_{u}=0,2 \mathrm{~T}_{1} ; 3-\tau_{u}=0,3 \mathrm{~T}_{1} ; 4-\tau_{u}=0,4 \mathrm{~T}_{1} ; 5-\tau_{u}=0,5 \mathrm{~T}_{1}$

Fig. 5 Experimental dependences of the amplitude of the first harmonic $(n=1)$ of the string vibration on the duration of the excitation impulse front $\tau_{f}$ for STMS (PLDS-150) at various values of $\tau_{u}$.

The materials presented in Fig. 5 show that:

1) The amplitude of the oscillations of the string has a nonlinear dependence on the duration of the excitation impulse front $\tau_{u}$ and its fronts duration $\tau_{f}$.

2) As the duration of the excitation impulse front increases, the amplitude of the vibration of the string y decreases. So for $\tau_{f}<0.5 \boldsymbol{T}_{1}$, i.e. at the maximum amplitude of the excitation impulse and the preservation of its trapezoidal form, the string oscillation amplitude drops by $45-50 \%$. 


\section{Discussion}

The experimental data of the dependence of the amplitude of the string vibrations on the duration of the fronts of the excitation impulse $\tau_{f}$, presented in Fig. (5), differ from the theoretical ones presented in Fig. (3). Thus, the values of the amplitude of the string vibrations obtained in the course of the experiment are lower than the theoretical values presented in Fig. (3), up to $26 \%$ at $\tau_{u}=0.5$ and $\tau_{f}=0.5 T$ and up to $45 \%$ at $\tau_{u}=0.2 T_{1}$ and $\tau_{f}$ $=0.5 T_{l}$.

This phenomenon is obviously due to at least three circumstances:

1) The theoretical dependence (1) was obtained for the case of equality of the values of the front and back fronts, i.e. symmetric shape of the excitation impulse. The actual shape of the excitation impulse during the experiment was different from the symmetric one, as shown in Fig. (4), namely, the leading front is $25-35 \%$ shorter than the trailing edge.

2) Oscillation amplitude of only the first harmonic y1 without taking into account highest harmonics $(n>1)$ was fixed while the energy of the excitation impulse was distributed between them.

3) During the experiment, it was not possible to fix the effect of the string oscillation precession, in the presence of which the oscillations amplitude will always be lower than the oscillations in one plane.

The experiment confirmed the general tendency of the string oscillation amplitude decrease when the duration of the excitation impulse front increases in the range up to $\tau_{\phi}$ $<0.5 T_{l}$. The decrease of the string vibration amplitude and, consequently, of the excitation of the EMF $\boldsymbol{e}$ induced in the electromagnetic coil results in the decrease of the "signal/noise" ratio at the output of the STMS and the instability of the whole monitoring system.

\section{Conclusions}

During the experimental investigations it was proved that in order to maintain the maximum amplitude of the string oscillations $\boldsymbol{y}_{\text {Max }}$ (provision the maximum "signal/noise" ratio) over the entire measurement interval $\left(\boldsymbol{t}_{i z m}\right)$, the amplitude of the excitation impulse of the STMS strings must be corrected in proportion to the duration of the excitation impulse fronts $\tau$, i.e. to the change of the parameters $\boldsymbol{C}$ and $\boldsymbol{R}$ of the «cable-switching equipment» system. These conditions ensure the stable oscillation of the SIPI strings and reliable operation of the STMS systems built on their basis. If these conditions are not fulfilled, STMS begins to give unstable readings soon after the introduction of the SMES systems into operation (from 1 to 5 years). So, they are excluded from the measurements as coming out of service and the informative basis for making decisions on the technical state of the high-rise buildings is reduced.

The experimental results obtained allow us to formulate future directions of the research:

- investigation of the influence of different durations of the front and back fronts of excitation impulses on the string oscillations amplitude;

- investigation of the influence of the precession of the string oscillation and the highest harmonics on the string oscillations amplitude;

- investigation of the ways of reducing the values of the parameters $\boldsymbol{C}$ and $\boldsymbol{R}$ of the «cable-switching equipment» system or their changes in time.

\section{References}


1. A. Zhivaev, G. Bolyrev, A System for Static and Dynamic Monitoring of Ice Sport Arena Text, IWSHM, 8th International Workshop on Structural Health Monitoring (Stanford, USA, September 2011)

2. L. Olson, Dynamic Bridge Substructure Evaluation and Monitoring, USA: US Department of Transportation, Federal Highway Administration, 219 (2005)

3. N. Sokolova, Sensors follows the safety. Monitoring as a pledge of quiet. St. Petersburg.: Building and City economy in St. Petersburg and region, 88 (2006)

4. D. Lysov, V. Guryev, V. Dorofeev, N. Nazjmov, Structural mechanics of engineering buildings and constructions, 2, 55-61 (2011)

5. D. Zavjalo, Engineering systems of high-rise buildings, High-rise buildings, 1, 107109 (2013)

6. A. Abdelrazak, High-rise buildings, 1, 96-103 (2016)

7. V. Gurje, V. Dorofeev, Unique and special technologies in buildings, 1 (2004)

8. TSN 31-332-2006 Residential and public high-rise buildings

9. MGSN 4.19-2005 Time standard and projecting principles of multifunctional high-rise buildings and residential complexes in Moscow

10. N. Kapustin, V. Tarakanovsky, A. Voznyuk, A. Klimov, Preventing of building and construction crash: Collected scientific articles. Issue, 9, 192-206 (2010)

11. CODE R 22.1.13-2013 "Safety in the state of emergency. Measures concerning civil defense, measures concerning prevention emergency state of natural and techno genic character. Structurizational monitoring system and control of the engineering systems of buildings and constructions. Requirements to the requence of building and exploitation"

12. N. Gusev, Significant problems of protection of inhabitants and territories from fires and catastrophes, (2006)

13. N. Gusev, V. Golovin, Sh. Iskhakov, V. Kishik, E. Pavlov, Scientific journal "Ber Board", 6 (2006)

14. STO 17330282.27.140.021-2008: Control-measuring systems and equipment of hydro-technical constructions of hydro-electric power stations. Arrangement of explaitation and technical maintenance. Standarts and requirements

15. Bernd Hiller, I. Sukhov, V. Lee, Automatized system of deformation monitoring (ASDM) at Sayano-Shushenskaya hydro-electric power stations, St. Petersburg: Hydrotechnique, 2, 12-15 (2015)

16. N. Gusev, Regional Informatics - 2006. Materials of the jubilee X International Conference. St. Petersburg, (2006)

17. SO 34.21.546-2003 (RD 153-34.2-21.546-2003) Regulations of creating and conducting natural observations and investigations at the dams out of soil materials, (2003)

18. L. Etkin, Vibro-frequency sensors. Theory and practice, (2004)

19. N. Gusev, Collection scientific articles. SPb, 21-34 (2007)

20. R. Zinoviev, Collected materials RUDN. Series "Engineering investigations", 2, 64-66 (2005)

21. N. Gusev, Materials of international scientific conference. St. Petersburg, (2006)

22. N. Gusev, Journal "Life and Safety", 3-4, 46-50 (2004)

23. STO 70238424.27.140.004-2008 Control-measuring systems and equipment of hydrotechnical constructions. Regulations of development. Standards and requirements

24. STO 70238424.27.140.025-2009: Hydro-electric power stations. Control-measuring systems and equipment of hydro-technical constructions. Metrological equipment and estimation of technical state and efficiency. Standards and requirements

25. R RSK 002-06 General requirements to the calibration methods used in Russian system of calibration 